\title{
Metodología de autocalibración para un sistema de sensores láser $2 \mathrm{D}$
}

\author{
Marta Torralba ${ }^{1}$, Lucía C. Díaz Pérez ${ }^{2}$, Margarita Valenzuela ${ }^{3}$, José A. Albajez ${ }^{2}$, José \\ A. Yagüe Fabra, \\ ${ }^{1}$ Centro Universitario de la Defensa, Zaragoza, Spain \\ ${ }^{2}$ Grupo de Ingeniería de Fabricación y Metrología Avanzada (GIFMA), \\ Instituto de Investigación en Ingeniería de Aragón (I3A) \\ Universidad de Zaragoza, Mariano Esquillor s/n, 50018, Zaragoza, Spain. \\ Tel.+34-976762707, e-mail: 1cdiaz@unizar.es \\ ${ }^{3}$ Universidad de Sonora, Departamento de Ingeniería Industrial, Hermosillo, México
}

\begin{abstract}
Este trabajo define un método de autocalibración para un sistema de sensores láser 2D a lo largo de un rango de trabajo de $50 \mathrm{~mm}$ x $50 \mathrm{~mm}$ con incertidumbre submicrométrica. Un encoder de malla 2D es usado como artefacto no calibrado. El correcto funcionamiento del método es comprobado, primero usando datos virtuales y luego experimentalmente.
\end{abstract}

\section{Introducción}

Una innovadora plataforma de nanoposicionamiento 2D (NanoPla) está en desarrollo con el objetivo de obtener una precisión submicrométrica a lo largo de un amplio rango de trabajo $(50 \mathrm{~mm} \times 50 \mathrm{~mm})$ [1]. El posicionamiento en $\mathrm{XY}$ está caracterizado por interferómetros de espejos planos, de la familia de láseres de Renishaw plc. Este trabajo trata con la definición de un método de calibración con el fin de obtener mejor precisión para el posicionamiento de la plataforma. Se ha definido un método de autocalibración ya que elimina la dificultad de encontrar un artefacto calibrado a escala nanométrica. Como artefacto no calibrado se usa un encoder de malla 2D. Este estudio está basado en un trabajo previo [2], que usa la misma configuración para el sistema de medida en 2D: interferómetros láser y un encoder de Heidenhain GmgH. En este abstract, primero se describe la configuración del sistema láser y el encoder usada para la toma de medidas. Después, se analiza el modelo matemático de medida del sistema láser. Seguidamente, se describe el algoritmo de autocalibración. Por último, el correcto funcionamiento del procedimiento se comprueba experimentalmente.

\section{Configuración experimental}

La configuración experimental para el procedimiento de autocalibración está representado en la Figura 1. El marco metrológico, fabricado en Zerodur, fue diseñado cuidadosamente con el fin de mejorar la precisión de los experimentos anteriores [2]. El mismo esquema ha sido usado en este estudio, aunque el enfoque y el modelo geométrico son distintos. El marco metrológico consiste en dos partes con movimiento relativo y para realizar el movimiento se usa un sistema de posicionamiento. Los espejos planos y el cabezal del encoder están sujetos a la parte superior del marco metrológico que a su vez está fijo al brazo del sistema de posicionamiento, que permanece estático. Los cabezales del láser y la base del encoder (placa graduada) están fijos a la parte inferior del marco metrológico, fijo, a su vez, a la mesa con desplazamiento horizontal del sistema, que es la que se mueve en el plano XY. Para está disposición, existe una desviación angular entre el sistema de referencia del sitema de posicionamiento y el del sistema láser $(\theta)$ y una desviación angular entre el sistema de referencia del sistema de posicionamiento y del encoder $(\sigma)$. El valor esperado para estos ángulos esta entre \pm 0.01 radianes.

\section{Modelo matemático del sistema láser 2D}

La definición del modelo matemático se ha hecho teniendo en cuenta la disposición de los espejos planos en la configuración experimental. Las relaciones geométricas están ilustradas en la Figura 2. El modelo asume que el haz incidente es coincidente con el reflejado, lo cual es coherente con las especificaciones dadas por el fabricante. Las relaciones geométricas están representadas en las Ecuaciones (1) a (4). $\mathrm{D}_{\mathrm{x}} \quad \mathrm{y} \quad \mathrm{D}_{\mathrm{y}}$ son los desplazamientos reales, a su vez $L_{x}$ y $L_{y}$ son las lecturas de los láseres en el eje $\mathrm{X}$ e $\mathrm{Y}$, respectivamente. 
$\mathrm{D}_{\mathrm{x}}=\frac{\mathrm{L}_{\mathrm{x}}}{\cos \left(\alpha_{\mathrm{xpitch}}\right)}$

$D_{y}=\frac{L_{y}}{\cos \left(\alpha_{\text {ypitch }}\right) \cos \left(\alpha_{x y}\right)}-\frac{\tan \left(\alpha_{x y}\right) L_{x}}{\cos \left(\alpha_{\text {xpitch }}\right) \cos \left(\alpha_{\text {ypitch }}\right)}$

Error in X-axis: $\delta_{\mathrm{x}}=\mathrm{D}_{\mathrm{x}}-\mathrm{L}_{\mathrm{x}}$

Error in y-axis: $\delta_{y}=D_{y}-L_{y}$

El método de autocalibración debe ser capaz de obtener incertidumbre submicrométrica en la medición dentro de los rangos esperados para los ángulos. Se espera que estos rangos sean, para $\alpha_{\mathrm{xy}}$ $\pm 5 \cdot 10^{-3}$ rad y para $\alpha_{\text {xpitch }} \mathrm{y} \alpha_{\text {ypitch }} \pm 15 \cdot 10^{-3}$ rad. Para obtener incertidumbre submicrométrica en el cálculo de $\mathrm{D}_{\mathrm{x}} \mathrm{y} \mathrm{D}_{\mathrm{y}}$, el ángulo $\alpha_{\mathrm{xy}}$ debe ser determinados con una incertidumbre $(\mathrm{k}=2)$ de $\pm 1 \cdot 10^{-6} \mathrm{rad}$, mientras que $\alpha_{x p i t c h} \mathrm{y} \alpha_{\text {ypitch }}$ requieren una incertidumbre $(\mathrm{k}=2)$ de $\pm 1 \cdot 10^{-3} \mathrm{rad}$.

\section{Procedimiento de autocalibración}

La autocalibración presenta muchas ventajas en aplicaciones de nanoposicionamiento [3]. Por lo tanto, se ha elegido como método para optimizar el comportamiento de la NanoPla en el área de trabajo de $\pm 25 \mathrm{~mm}$. Son necesarias dos vistas del encoder para el proceso, la vista inicial (Vista 0) y la rotada (Vista 1), que se consigue rotando el encoder 180 grados alrededor del eje Z. Los errores globales determinados por esta técnica $\left(\mathrm{V}_{0}\right)$ son expresados como la suma de los errores de posicionamiento del sistema laser $\left(\mathrm{M}_{0}\right)$, los del artefacto no calibrado $\left(\mathrm{E}_{0}\right)$ y los errores de alineación del artefacto no calibrado, en cada vista. Los datos de entrada del algoritmo son las lecturas del sistema laser y del encoder, en la misma posición. Las lecturas del encoder son alineadas matemáticamente con el sistema de referencia del sistema láser $(\theta-\sigma)$, por lo tanto los errores de alineación se cancelan. La suma global de errores queda representada en la Ecuación 5, para la Vista 0.

$\mathrm{V}_{0}\left(\mathrm{x}_{\mathrm{i}}, \mathrm{y}_{\mathrm{j}}\right)=\mathrm{M}_{0}\left(\mathrm{x}_{\mathrm{i}}, \mathrm{y}_{\mathrm{j}}\right)+\mathrm{E}_{0}\left(\mathrm{x}_{\mathrm{i}}, \mathrm{y}_{\mathrm{j}}\right)$

$\left(\mathrm{x}_{\mathrm{i}}, \mathrm{y}_{\mathrm{j}}\right)$ representa el punto de medición, la malla tiene $\mathrm{NxN}$ puntos, por lo que $\mathrm{i}, \mathrm{j}=1,2, \ldots, \mathrm{N}$. En este caso, en el área de trabajo de $50 \mathrm{~mm}$ x $50 \mathrm{~mm}$, se han medido $11 \times 11$ puntos. Por lo tanto, se tienen 121 lecturas del sistema láser y del encoder en cada vista. Es posible asumir que los errores del artefacto no calibrado (el encoder) pueden ser cancelados relacionando las ecuaciones de la Vista 0 y la Vista 1 Combinando las Ecuaciones (3), (4) y (5), se obtienen las siguientes expresiones para la Vista 0 y la 1 en los ejes $\mathrm{X}$ e $\mathrm{Y}$.

$$
\begin{aligned}
& V_{0 x}\left(x_{i}, y_{j}\right)=\delta_{x}\left(x_{i}\right)+E_{x}\left(x_{i}, y_{j}\right) \\
& V_{0 y}\left(x_{i}, y_{j}\right)=\delta_{y}\left(x_{i}, y_{j}\right)+E_{y}\left(x_{i}, y_{j}\right) \\
& V_{1 x}\left(x_{i}, y_{j}\right)=\delta_{x}\left(x_{i}\right)+E_{x}\left(-x_{i},-y_{j}\right) \\
& V_{1 y}\left(x_{i}, y_{j}\right)=\delta_{y}\left(x_{i}, y_{j}\right)+E_{y}\left(-x_{i},-y_{j}\right)
\end{aligned}
$$

Representando $\delta_{\mathrm{x}} \mathrm{y} \delta_{\mathrm{y}}$ en función de las ecuaciones (1) y (2) y combinando las ecuaciones de las distintas vistas es posible obtener $\alpha_{\mathrm{xy}}, \alpha_{\mathrm{xpitch}} \mathrm{y} \alpha_{\mathrm{ypitch}}$, en función de las lecturas tomadas. Antes de iniciar las medidas experimentales, el correcto funcionamiento del algoritmo ha sido verificado con datos virtuales, para valores de $\alpha_{x y}, \alpha_{\text {xpitch }} \mathrm{y} \alpha_{\text {ypitch }}$ dentro de los rangos esperados.

\section{Autocalibración experimental del sistema láser}

La autocalibración empieza con el establecimiento de una longitud de referencia para el sistema a calibrar. En este caso se usan los ejes centrales X e Y del encoder, que han sido calibrados por el fabricante. Cuando ya se han medido todos los puntos, se aplica el algoritmo de calibración y se obtienen los valores de $\alpha_{\mathrm{xy}}, \alpha_{\text {xpitch }} \mathrm{y} \alpha_{\text {ypitch }} \mathrm{y}$ la desviación angular entre el sistema de referencia del sistema láser y el encoder, para la Vista $0\left(\theta-\sigma_{0}\right)$, y la $1\left(\theta-\sigma_{1}\right)$. Los resultados obtenidos están representados en la Tabla 1 , los valores están dados con la incertidumbre requerida y están dentro del rango esperado. Las lecturas del láser $\left(\mathrm{L}_{\mathrm{x}} \mathrm{y} \quad \mathrm{L}_{\mathrm{y}}\right)$ tienen que ser corregidas sustituyendo los valores de $\alpha_{\mathrm{xy}}, \alpha_{\mathrm{xpitch}} \mathrm{y} \alpha_{\mathrm{ypitch}}$ en las ecuaciones (1) y (2) para obtener los desplazamientos reales $\left(\mathrm{D}_{\mathrm{x}} \mathrm{y} \mathrm{D}_{\mathrm{y}}\right)$. Con las lecturas corregidas del sistema láser, se ha calculado el error de medición del encoder en los ejes centrales $\mathrm{X}$ e $\mathrm{Y}$. Posteriormente, se ha comparado con el error dado por el fabricante en su certificado de calibración. Los dos errores son muy similares lo que permite afirmar que el método propuesto es adecuado.

\section{Conclusiones}

Se ha realizado la autocalibración de un sistema de sensores laser 2D para una plataforma de nanoposicionamiento a lo largo de una amplia área, usando un encoder 2D como artefacto no calibrado. Este estudio demuestra la idoneidad de este procedimiento. En trabajos posteriores se ha realizado la calibración del encoder usando el 
sistema láser ya calibrado y se ha calculado la incertidumbre de la calibración.

\section{Agradecimientos}

Este trabajo ha sido financiado por el proyecto DPI2010-21629-C02-01 "NanoPla" and DGA-FSE del gobierno de España. El programa FPU del MECD ha patrocinado a la segunda autora y el Consejo Nacional de Ciencia y Tecnología (CONACYT) del gobierno de México a la tercera autora.

\section{REFERENCIAS}

[1]. TORRALBA, M. et al. Design Optimization for the Measurement Accuracy Improvement of a Large Range Nanopositioning Stage. En: Sensors. 2016, no. 16(1), pp. 84

[2]. YAGÜE FABRA, J.A., VALENZUELA, M., ALBAJEZ, J.A. and AGUILAR, J.J. A thermallystable setup and calibration technique for 2D sensors. En: CIRP Annals Manufacturing Technology. 2011, no. 60, pp. $547-55$

[3]. JEONG, Y. H., DONG, J. and FERREIRA, P. M. Self-calibration of dual-actuated single-axis nanopositioners. En: Measurement Science and Technology. 2008, no. 19(4), pp. 045203

\section{Figuras y Tablas}

Figura 1. Configuración experimental para la calibración

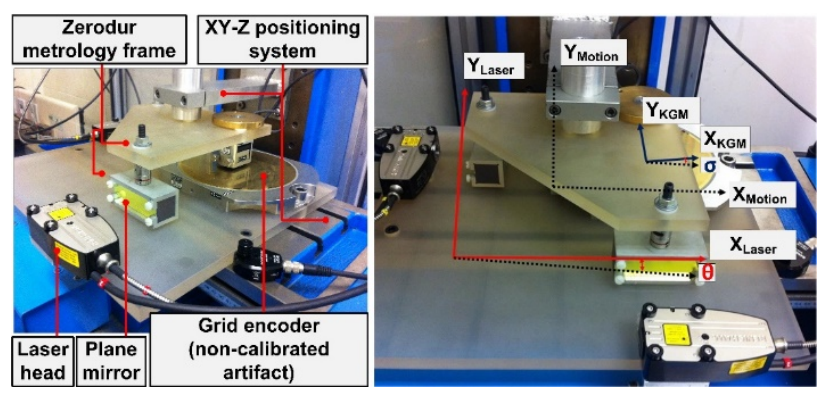

Figura 2. Modelo geométrico del sistema láser

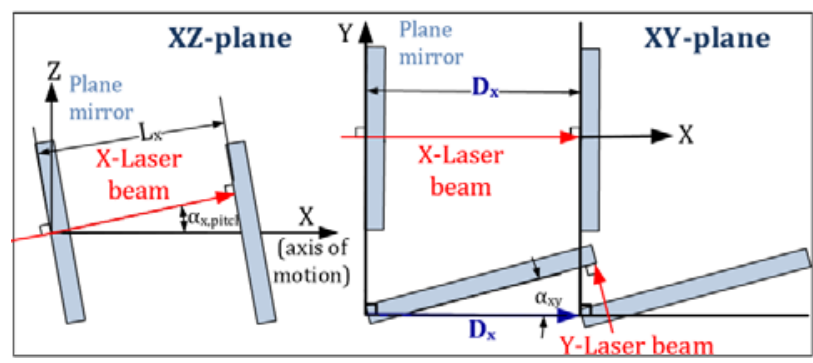

Tabla 2. Parámetros geométricos obtenidos en la calibración

\begin{tabular}{|l|l|l|l|}
\hline Parámetro & $\begin{array}{l}\text { Rango } \\
{[\mathrm{rad}]}\end{array}$ & $\begin{array}{l}\text { Incertidumbre } \\
{[\mathrm{rad}]}\end{array}$ & $\begin{array}{l}\text { Resultado } \\
{[\mathrm{rad}]}\end{array}$ \\
\hline$\alpha_{\mathrm{xy}}$ & $\pm 5 \cdot 10^{-3}$ & $\pm 1 \cdot 10^{-6}$ & -0.003920 \\
\hline$\alpha_{\mathrm{xpitch}}$ & $\pm 12 \cdot 10^{-3}$ & $\pm 1 \cdot 10^{-3}$ & 0.011 \\
\hline$\alpha_{\text {ypitch }}$ & $\pm 12 \cdot 10^{-3}$ & $\pm 1 \cdot 10^{-3}$ & 0.004 \\
\hline$\theta-\sigma_{0}$ & \pm 0.02 & $\pm 1 \cdot 10^{-4}$ & -0.0049 \\
\hline$\theta-\sigma_{1}$ & \pm 0.02 & $\pm 1 \cdot 10^{-4}$ & -0.0046 \\
\hline
\end{tabular}

